\title{
Experiences of Community Members on Reporting Community Maternal Deaths in Mangochi District of Malawi
}

\author{
Jane Dzoole1, Mercy Pindani2 ${ }^{*}$, Alfred Maluwa² \\ ${ }^{1}$ Kamuzu Central Hospital, Ethel Muthalika Maternity Wing, Lilongwe, Malawi \\ ${ }^{2}$ Kamuzu College of Nursing, University of Malawi, Lilongwe, Malawi \\ Email: ${ }^{*}$ mercypindani@kcn.unima.mw
}

Received 6 March 2015; accepted 21 March 2015; published 27 March 2015

Copyright (C) 2015 by authors and Scientific Research Publishing Inc.

This work is licensed under the Creative Commons Attribution International License (CC BY). http://creativecommons.org/licenses/by/4.0/

(c) (i) Open Access

\section{Abstract}

The purpose of this study was to explore and describe the experiences of community stakeholders on reporting community maternal deaths to relevant authorities in Mangochi District of Malawi. The study employed qualitative hermeneutic phenomenology approach to data collection, analysis and interpretation. It was conducted in three health zones of Mangochi district which are Monkey-bay, Mangochi boma and Namwera zones. Purposive sampling was used to select major community stakeholders on issues of safe motherhood and these were; Village heads, Health Surveillance Assistants (HSAs), safe motherhood volunteers and members of village health committees (VHCs). A total of eighteen in-depth interviews and three focus group discussions were conducted. Descriptive statistics were computed for the demographic variables and the qualitative data were analysed using modified Colaizzi (1978) method based on Heideggerian and Gademerian philosophy. Findings showed that community maternal deaths were not always reported because there were no records in the district. Most participants lacked knowledge on the process and their role in reporting community maternal deaths despite knowing the importance of reporting such deaths. However, findings indicated a positive perception of participants towards reporting community maternal deaths to authorities. The study recommends that health education be offered to community members and Health Surveillance Assistants regarding reporting maternal deaths to improve the situation.

\section{Keywords}

Community Maternal Deaths, Community Members, Reporting, Safe Motherhood, Maternal Death Surveillance and Response, Maternal Health

\footnotetext{
${ }^{*}$ Corresponding author.

How to cite this paper: Dzoole, J., Pindani, M. and Maluwa, A. (2015) Experiences of Community Members on Reporting Community Maternal Deaths in Mangochi District of Malawi. Open Journal of Nursing, 5, 226-236. 


\section{Introduction}

Childbirth is meant to be a universally celebrated event and an occasion for cheerful making. Yet, for many thousands of women each day, child bearing is experienced not as the joyful event, but as unpleasant hell that may end in death [1]. Worldwide 536,000 women die each year from complications of pregnancy and childbirth [2]. Ninety nine percent of these deaths occur in developing countries. Slightly, more than half of the maternal deaths occur in the sub-Saharan Africa region followed by South Asia which account for $86 \%$ of global maternal deaths. Maternal mortality ratio is highest in developing regions at 240 per 100,000 births versus 16 per 100,000 in developed countries [3].

World Health Organisation [3] defines maternal death as the death of a woman while pregnant or within 42 days of termination of pregnancy, irrespective of the duration and site of the pregnancy, from any cause related to or aggravated by the pregnancy or its management but not from accidental or incidental causes. According to 2010 global estimates maternal mortality ratio is at 210 [4]. Although there has been a drop in maternal deaths worldwide, the global maternal mortality ratio is far from meeting the annual decline of $5.5 \%$ required to achieve Millennium Development Goal (MDG) 5 of improving maternal health.

Most maternal deaths are avoidable and the value of audits surrounding reduction of maternal death need not be overemphasised [5]. However, insufficient data collection and reporting system are big challenges [4] [6]. Nieburg [4] recommends the need to improve the existing data gap in order to conduct death reviews.

Malawi is a low income country with a maternal mortality ratio estimated at 675/100,000 live births [7]. Most of these deaths are audited at facility level [8] [9]. However, most maternal deaths that occur in the community are not audited because they are not reported to relevant authorities.

Since the late 1980s, improving maternal health and reducing maternal mortality have been key concerns of several international summits and conferences including the Millennium Summit [10]. Global efforts to address maternal mortality started with the Safe Motherhood Initiative in 1987. The problem was further highlighted during the 1994 International Conference on Population and Development and the Women Deliver conferences held in 2007 and 2010. In 2000, the United Nations included the reduction of maternal mortality by $75 \%$ by 2015 as one of the 8 Millennium Development Goals (MDG 5), [11]. The MDG5 calls for improving maternal health of which maternal mortality ratio is a key indicator for monitoring progress towards its achievement. One of the strategies to improve maternal health as recommended by WHO [12] is by conducting maternal death audit. Maternal death audit is an in-depth systematic review of maternal deaths to delineate their underlying health social and other contributory factors, and the lessons learned from such an audit are used in making recommendations to prevent similar future deaths [13]. The process of maternal death audit empowers local authorities to understand and take steps to improve maternal health. However, many pregnancy-related deaths especially those that happen in the community go unnoticed by relevant authorities because they are not reported. Hence, there are substantial errors in the estimates of maternal mortality. In addition, comprehensive measures to address the problem are not taken despite the fact that facility-based and community-based maternal death reviews are strongly endorsed as educational process for both health workers and community members.

Malawi adopted a combination of facility and community based maternal death audits to improve maternal health as stipulated in a road map for the reduction of maternal deaths [14]. Facility based maternal death audits have thus been institutionalised in most hospitals [8]. However, there is scanty information regarding community maternal death and community maternal death audit. Unofficial results indicate that Mangochi had 62 community based maternal deaths from December 2011 to November 2012. A study by Hofman and Ndemera [15] in Nankumba area of Mangochi district showed that $44 \%$ of maternal deaths occurred outside health facilities. Similarly Ochi, [16] in her study conducted in Kaduna state in Nigeria reported a significant number of maternal deaths that occurred at home in rural areas. However, these maternal deaths are neither reported nor registered at District Health Office. These results entail that community maternal health is not comprehensively addressed. Reporting each and every maternal death that occurs in the community is one of the interventions that would contribute towards the reduction of premature death. Therefore, community participation is fundamental in order to report community maternal death to achieve the MDG 5 of improving maternal health [17]. The purpose of this study, therefore, was to explore and describe experiences of selected community members on reporting community maternal deaths in Mangochi district of Malawi.

\section{Methodology}

The study employed qualitative hermeneutic phenomenology as a research method. This approach was chosen 
because it produces insights into human experience [18] and data was collected at one point only in time. The methodology enabled the exploration of participants' experiences with further abstraction and interpretation by the researchers based on theoretical and personal knowledge. The qualitative research methodology enabled a systematic, subjective approach to describe life experiences and situations and give them meaning [19]. The setting for the study was Mangochi district which is in the Southern part of Malawi. Purposive sampling method was used to select eighteen participants who took part in face to face in-depth interviews and in the three focus group discussions.

\subsection{Inclusion Criteria}

To be recruited for the study, participants had to be village heads, Health Surveillance Assistants (HSA) or members of health committees and safe motherhood volunteers who experienced community maternal deaths in their area of responsibility for the past three years. They were also expected to show willingness to participate and share their experiences in the study.

\subsection{Ethical Issues}

Ethical approval to conduct the study was obtained from College of Medicine Research and Ethics Committee (COMREC). Permission to conduct the study in Mangochi district was sought from the District Commissioner (DC) and the District Health Officer (DHO) for Mangochi. Participation was voluntary and informed consent was obtained from each participant who met the inclusion criteria after explaining the nature and purpose of the study. The participants were allocated code numbers instead of names to ensure confidentiality and anonymity.

\subsection{Data Collection}

The research team comprised the principal investigator, and one research assistant. The research assistant was a community health nurse/midwife registered with nurses and midwifes council with ability to read and speak English, Chichewa and Chiyao. The training of research assistant for data collection was for a day and data was collected in the month of November 2013. Eighteen (18) in depth interviews and three (3) focus group discussions were conducted. The participants were identified from the three health zones of Mangochi district namely; Monkey-bay, Mangochi boma and Namwera zone. Participants that met the inclusion criteria and consented to participate in the study were interviewed at a private place. All the interviews and discussions were carried out in Chichewa, because all participants were able to communicate in Chichewa. During data collection, each participant was engaged in a dialogue using an open ended interview guide. Probes were made to understand the participants' responses and comments on an issue under discussion. The discussions concentrated on their existing practices of reporting community maternal deaths, their knowledge of the process and importance of reporting maternal death at community level, their perceptions and factors that influence them to report maternal deaths at community level. A digital recorder was used to record the discussions and field notes were manually taken to aid the data collection process. Interviews lasted between 45 and 60 minutes.

\subsection{Data Analysis}

Data analysis was done simultaneously with data collection. Descriptive statistics were computed for the demographic variables and the qualitative data was analysed using modified Colaizzi [20] based on Heideggerian and Gademerian philosophy [21]. The modification is based on Colaizzi’s failure to portray role of reflection in enabling the researcher to develop the meanings of the phenomena being investigated. Husserl's philosophy emphasizes the importance of bracketing "researchers to put aside their preconceptions of the phenomena being studied”. However, Heidegger and Gadamer [21] greatly uphold the importance of pre-understanding in facilitating understanding of the phenomena being investigated, hence the modification. Steps of analysis involved description of the experiences by participants which was accomplished through conversational interviews. Verification of the descriptions to the participants was done immediately and data were transcribed verbatim to develop phenomenological text. The transcriptions were done by the researcher to ensure accuracy. The texts were then translated into English. Clusters of themes from the formulated meanings were identified and these themes are reported as the study findings. 


\section{Results}

\subsection{Demographic Characteristics of the Participants}

Most of the participants $(50 \%, n=9)$ were aged above 41 years, while $27.8 \%(n=5)$ were between the ages of 21 - 30 and 22.2\% $(n=4)$ were between 31 - 40 years. There were more female $(55.6 \%(n=10)$ than male $(44.4 \%$ $(\mathrm{n}=8)$ participants. Christians comprised 55.6\% $(\mathrm{n}=10)$ of the participants and $44.4 \%(\mathrm{n}=8)$ were Moslems. Most participants, $80 \%(n=12)$ were literate and numerate and 55.6\% $(n=10)$ of them were able to speak both Yao and Chewa while 44.4\% $(n=8)$ were able to speak Chewa only. Most of participants 94.4\% ( $\mathrm{n}=17) \mathrm{had}$ stayed or worked in the area for more than five years. Only 5.6\% $(n=1)$ had stayed or worked for one to two years. Fifty percent $(n=9)$ of participants interviewed were Yao, 38.7\% $(n=7)$ Chewa, 5.1\% $(n=1)$ Lomwe and $5.1 \%(n=1)$ belonged to other tribes. Most study participants had low level of education. There were equal numbers of participants $27.8 \%(n=5)$ with junior primary, senior primary and no education. However, $16.7 \%$ $(n=3)$, had senior secondary education.

\subsection{Emerging Themes}

Four major themes emerged from the participants' narrations. The themes were: the practice of reporting community maternal deaths, records of community maternal deaths, knowledge of reporting community maternal deaths and perceptions of participants on reporting community maternal deaths.

\subsubsection{Reporting Community Maternal Deaths}

The participants' narrations show that community maternal deaths are not taken as a tragedy that must be notified to relevant authorities. Most participants indicated that community maternal deaths were handled like any other death. Almost all village heads indicated that after receiving reports of community maternal death from family members, they only informed village members just like any other death. No reports were sent to hospitals. This was reflected by participant \# 15:

"You mean what I did? I informed the sheikh and other influential people in the village. I told them that they should tell all people in the village that the woman died due to motherhood and they should not hide anything. I did not report to anyone at the hospital. I think no one reported to the hospital too".

However, most participants stated that village heads are always the first people to be notified of community maternal death by family members of the deceased just like any other death in the village. One village head (participant \# 9) had this to say: "when I learnt about this death from the deceased family members, I informed all people in the village just like any other death and I don't know if it was reported to the hospital".

Participant \# 7 also stressed: "As safe motherhood committee members, we are supposed to inform the village head about any maternal death but I am not sure if any member of safe motherhood committee informed the village head about this maternal death".

Majority of the village heads said that the main reason for informing all people in the village is for them to be present during the funeral ceremony. "We inform people so that they can be present during the funeral ceremony, otherwise there would be no people at the ceremony". Additionally, village heads do not specifically inform HSAs responsible for the area about the community maternal death. All HSAs indicated that they learnt of the community maternal death from other people just like any other death. Participant \# 12 stated: "During this time I just learnt from people who were discussing amongst themselves that a certain woman had died at the traditional birth attendant although she had delivered at the hospital”. It was noted that although village heads do not specifically inform HSAs about the community maternal deaths, most of the HSAs sometimes know about the death because they are able to assist external researchers who require such information. One participant had this to say: "A certain lady from Lilongwe who was conducting a research study and said she was doing school outside the country came to find out more about this death. She came with mayi Abudu (Mrs Abudu) our HSA. After asking us some questions she never came back and no one else came again apart from you”.

It was noted from the responses that when there is a maternal death, people in the community are afraid. "It was a first time that a woman had died in this village due to motherhood. It was very sad and everyone was afraid because this woman delivered at home and everywhere people were talking. I learnt of this death from people in the village as they were discussing. I did not do anything. I am not sure if hospital staff were informed". 
All participants indicated that they did not know they were supposed to report community maternal death to the hospital. They stated that lack of knowledge was the main factor that prevented most participants from reporting community maternal death as indicated by participant \# 1. "What prevented me to report this maternal death is basically lack of knowledge and I also did not know who to report to at monkey-bay". Furthermore, most participants indicated that they were not told to report community maternal death to the hospital as narrated by participant \# 16. "I did not report the death to the hospital because I have not been told to report such deaths to the hospital". Some HSAs indicated that they did not report because of unavailability of village registers as highlighted by Participant \# 12. "I did not do anything because at this time we were not given village registers. I could have recorded the death in the village register". Participant \# 18 also added by saying that: "No, I did not report to the hospital because at that time we were not told to record in the village register".

All the participants stated that their culture and religion had no influence on reporting community maternal death to relevant authorities in their area as narrated by participant \# 1 .

"According to my culture and my religion there is no problem with reporting community maternal death, actually it could be encouraged. But may be the religion of the person who has died would prevent so that people don't know that the woman has died because of the motherhood".

\subsubsection{Records of Community Maternal Death}

Findings revealed that community maternal death records were not available in the communities in Mangochi district. Almost all participants (17 out of 18 ) indicated that they did not have any record of community maternal death in their area. "No, we do not have those records, may be the group village head has because at first we used to give her reports but it was stopped because she did not know what to do with the reports" (participants \# 6). Another participant also said: "In this village, we do not have any type of safe motherhood records". However, one village head mentioned that records were available although she could not trace where she kept them since she moved from her old home. "I have safe motherhood records and members of safe motherhood committee also keep records. Since I moved into another house I cannot trace where I kept them. We record name of woman, date of death and cause of death.” (Participant \# 3).

\subsubsection{Knowledge on Reporting Community Maternal Death}

Almost all participants gave responses that indicated inadequate knowledge on the process of reporting community maternal death and on their role on reporting community maternal death. However, participants showed some knowledge of the importance of reporting and consequences of not reporting community maternal deaths to relevant authorities. Findings also showed that all participants were not trained in reporting community maternal deaths.

"You mean the process of reporting community maternal death? Aaa! Is there a specific process? I really don't know that one. Is it different from any other death? But let me just say I do not know it.” (Participant \# 7).

Another participant \# 5 stated: "Honestly, I do not know any process of reporting community maternal death because I was not told". One HSA (Participant \#18) also stated that "I know that every death that takes place in the village is supposed to be recorded in village registers but about the process, I do not know anything". Other participants mentioned that the process was similar to that of any other deaths that take place in the community: "The process is like any other death. There is no difference".

Findings also revealed that participants knew the consequences of not reporting community maternal deaths to relevant authorities.

"If it is not reported, it means hospital staff cannot come to do maternal death audit". Women will continue to be delivering at home or at the traditional birth attendants. They will continue to deliver at home and we will have more and more maternal deaths. If it is not reported, the cause of death for this particular woman cannot be known".

In spite of some knowledge of the importance of reporting community maternal deaths and consequences of not reporting community maternal deaths, the findings show that majority of the participants were not aware of their role in reporting community maternal death. Almost all village heads indicated that their role is to inform all people in the village. "My role is to inform all people in this village and give proper counselling where things went wrong” (participants \# 9, 14 and 15). 
One participant however indicated that her role was to inform HSA in the village about maternal death "My role is to inform the Health Surveillance Assistants and all people in this village". Some health surveillance assistants explained that their role was to educate people so that they can be able to prevent further deaths and also to record in the village register and send biannual reports as stated by Participant \# 18: "My role is to educate people so they can be able to prevent further maternal death. To the hospital, my role is to record in village register and send reports to my supervisor biannually". Majority of village health committee members and safe motherhood committee members said that they did not have any role. "I don't have any role in reporting community maternal death but relatives of the deceased are supposed to inform the village head and the village head informs all people in the village" (participants \# 6). One member of village health committee (participant \# 13) explained further as follows: "As a village health committee member, I don't have any role in maternal death because in this village we have a safe motherhood committee that deals with issues of safe motherhood. Members of that committee should have a role otherwise it will seem like we are monopolising activities".

\subsubsection{Training on Reporting Community Maternal Death}

Majority of participants were not trained in community maternal death surveillance and response. "I have never been trained on reporting community maternal death" (Participants 1, 12 and 18). One health surveillance assistant (participant \# 1) further explained that during their basic training they covered something on safe motherhood but they did not learn on reporting community maternal death. "No, I have not attended any safe motherhood training, but during my basic HSA training, we learnt a bit about safe motherhood like focused antenatal clinics and to encourage mothers to deliver at the hospital" Similarly, one member of safe motherhood committee (participant \# 5) reported; "I was trained in safe motherhood some time back but I did not learn anything on reporting community maternal death".

\subsubsection{Perceptions on Reporting Community Maternal Death}

Findings demonstrated that there were both positive and negative perceptions on reporting community maternal deaths. Majority of participants (17 out of 18) explained that it is important to report all community maternal death so that maternal death audit should be done since such deaths are not audited. "I think it is important that all maternal deaths that occur in the community must be reported. They do not know that there has been a maternal death in the community. For hospital staff to know, it is important that they should be informed of any maternal death that happens in the community (Participant \#18).

One of the village health committee members (participant \# 8) said: "They say 'no woman should die while giving birth'. This means that maternal death is preventable. If it is so, whenever we have maternal death, it is important that it is known to authorities at the hospital because they are the ones who can be in a position to give proper counselling to prevent further deaths". Some participants suggested that community maternal death should be reported by HSA because more often they are aware of almost all the deaths in the village. "I personally think hospital staff should be informed of any maternal death through the HSAs. Usually HSAs know of almost all the deaths that occur in the village so they are supposed to report to the hospital". This was concurred by one of the HSAs (Participant\# 18) although he denied of usually being aware of all the deaths. "As HSA's we can be informing responsible people at the hospital. But, there is need for coordination between village heads and HSA's because sometimes we don't know all the deaths that occur in the village. It is important that the village heads through safe motherhood committee members inform us as soon as possible."

On a negative note, one member of safe motherhood committee indicated that all deaths are equal and must be treated as such since life of a person has been lost. This fact was explained by participant \# 3 as follows; "All deaths are equal, whether one dies of malaria or motherhood, they should be treated equal. All necessary arrangements should be similar because life of a person has been lost. If the death can be prevented, the most important thing is to ensure that measures are put in place so that a similar death in similar situation does not happen again. If it means reporting to the hospital all deaths must be reported to the hospital".

The study findings indicated that training, coordination and routine reporting would improve community maternal death reporting. Responses from all participants and all the focus group discussions indicated that training would improve reporting of community maternal deaths as explained by one of the village heads: "It means that all chiefs should be trained on this. May be it is because I have not been trained in safe motherhood (Participant \# 14). One of the HSAs (participant \# 12) stated; "All HSAs need to be trained because most of us do not". Participant \# 13 stated that; "May be it is also important to have coordination between village health committee mem- 
bers and safe motherhood committee members so that everyone participates and no one monopolizes all activities".

\section{Discussions of Findings}

\subsection{Reporting Community Maternal Death}

Results show that most community members have never reported community maternal deaths to relevant authorities in Mangochi district. The findings are in agreement with the findings of Munjanja et al., [22] in a study conducted in Zimbabwe on the assessment of notification of maternal deaths. They noted gross under-reporting of maternal deaths at the district, community, provincial and tertiary levels. Likewise, in the Gaza Strip, a descriptive retrospective review of maternal mortality was done where one of the limitations of the study was that not all deaths were made known to relevant offices, as such some maternal deaths were missed [23].

In this study, lack of knowledge was identified as the main reason for not reporting. All participants indicated that they did not know they were supposed to report community maternal death to the hospital. Furthermore, most participants indicated that they were not told to report community maternal deaths to the hospital. The findings are in agreement with Ochi [16] in a study on quality of health care services and maternal mortality in rural communities in Kaduna where ignorance was one of the factors that influenced action regarding seeking maternal health care service by the community. The findings are however, contrary with those reported by Kalaichandran et al., [24] who assumed that much of this underreporting was perhaps due to the stigma and feared percussions associated with reporting such deaths. Some factors associated with non-reporting could be; unavailability of post-mortem examination, deaths in the first trimester of pregnancy, and time interval between pregnancy termination and death [25].

Some HSAs indicated that they did not report because of unavailability of village registers, but it is unlikely that unavailability of village registers can prevent one from reporting community maternal deaths as they are used to document information about the number of people living in a village and new births and deaths. These HSAs might have responded in relation to recording. It was revealed that culture and religion did not have negative influence on reporting community maternal deaths to relevant authorities. Findings disagree with Ochi [16] who states that cultural factors influence action regarding seeking maternal health care service by the community.

\subsection{Records of Community Maternal Deaths}

Findings revealed that there are no community maternal death records in Mangochi district despite acceptance by community members that there have been such deaths in their areas. This is in agreement with findings of Ochi [16] in a study conducted in Kaduna state in Nigeria who also noted that a significant number of maternal death went unrecorded because they did not occur in a health facility. Similarly, no data were found on how many deaths had occurred at home or in transit to or from facilities in Nigeria. In Malawi, although record keeping in referral and other hospitals are emphasized, these practices are poor. There are a lot of underreporting observed in institutions where record-keeping is theoretically in place, it may therefore be expected that direct and especially indirect maternal deaths in the community would be underreported to a greater extent.

Maternal death records serve to describe the incidence, prevalence, and causes of maternal deaths as well as the availability and effectiveness of attempts to prevent them [26]. Unavailability of community maternal death records indicates that the magnitude of maternal mortality both at district and at national level is underestimated. Furthermore, it means comprehensive actions to mitigate the problem are not taken hence the saying "no woman must die during child birth" is just lip service. In order to avoid further community maternal deaths and improve maternal health, all community maternal deaths should be identified, reported, reviewed, recorded and responded to accordingly. Additionally, the systematic recording of maternal death is an important routine activity to help in tracing and assessing the progress a country is making towards reaching the MDG 5 target.

\subsection{Knowledge on Reporting Community Maternal Deaths}

Findings also revealed that community members and HSAs have poor and inadequate knowledge on reporting community maternal deaths. Responses of participants showed that they had poor knowledge of the process and 
their role in reporting community maternal deaths. Almost all participants mentioned that they did not know the process and others said that the process of reporting was just like any other death. It was also noted that majority of the responses indicated that participants were not aware of their role in reporting community maternal death. Almost all village heads indicated that their role is just to inform all people in the village. Only one village head indicated that her role was to inform HSAs responsible for the village. Additionally, HSAs indicated that their role was to educate people so that they can be able to prevent further deaths. They also indicated that their role was to record in the village register and send biannual reports to their supervisors. However, this was not actually done. It was also noted that majority of village health committee members thought that they did not have any role in reporting community maternal deaths. In Malawi, although the road map for accelerating the reduction of maternal and neonatal mortality and morbidity and the guidelines for community initiatives for reproductive health highlights on recording and reporting maternal health outcome in the community, they do not clearly state where to report [27]. It is therefore not surprising that both community members and HSAs have poor knowledge of the process and their role in reporting community maternal deaths.

On a positive note, the participants indicated to have some knowledge regarding the importance of reporting community maternal deaths. Participants were able to mention benefits such as: to prevent further deaths; to make future plans; to do maternal death audits; to stop women to deliver at the TBAs and to provide proper counselling. The fairly good knowledge from participants on importance of reporting could be attributed to mass media communication especially the radio. According to Kululanga et al., [28], channels that use mass media are able to reach wide audience, can convey simple information and can be a powerful force in mediating social norms. Notifying maternal death can enhance maternal death surveillance and improve response mechanisms to avoid future deaths. Most countries have introduced action-oriented review mechanisms following maternal death notification. The review mechanisms require analysis of the circumstances of each death, identification of avoidable factors and action to improve care at all levels of the health system, from home to hospital [29]. Therefore, active community engagement for maternal death especially those that occurs in the community is needed to ensure that the circumstances surrounding each death are fully elucidated and that there are comprehensive and feasible recommendations for follow-up action.

Results further show that community members and HSAs were not trained in community maternal death surveillance and response, especially on reporting community maternal deaths. Mairiga et al., [30] in their study, stressed the importance of community mobilization and advocacy because a good number of community members had poor knowledge of maternal mortality. Community mobilisation and advocacy are important factors in the utilization of primary health services. Community mobilisation and advocacy also help to promote ownership and sustainable improvement in the health services [31].

\subsection{Perception on Reporting Community Maternal Deaths}

Despite mixed perception on reporting community maternal deaths, majority of participants (17 out of 18) expressed good perception and had a positive opinion regarding reporting maternal mortality. This finding is important to note because this study was conducted in rural areas where most participants (83.4\%) had no education or very low education. The findings are however in contrast with those reported by Lawoyin et al., [32], who carried out a cross-sectional, community-based study to assess men's perception of maternal mortality in Nigeria and found that efforts were required to improve men's attitudes in order to make them active participants in the fight to reduce maternal mortality. Both men and women in this study expressed good attitude toward reporting community maternal death to relevant authorities. However, this did not influence their decision to report community maternal death.

Several views came out on best ways to report community maternal deaths. Most participants expressed that it is important for health personnel to be informed about community maternal death but could not explain how these health personnel should be informed of community maternal death. Majority stated that HSAs should be responsible for reporting the deaths to the hospital. Training, routine reporting and coordination were factors that were identified to improve reporting of community maternal death in the district.

\section{Study Limitations}

This study is part of an academic requirement and had to be completed within a stipulated period. Time was 
therefore a limiting factor. In addition, as a scholar with financial constraints, the study could not have covered much wider areas than the ones stated, therefore the findings cannot be generalised to the whole nation of Malawi.

\section{Recommendations}

Clear standards and guidelines for reporting community maternal deaths should be made available by Ministry of health, through Reproductive health unit. The standards and guidelines should be communicated to all district health offices in the country. The District Health Offices should orient all health workers and all community stakeholders responsible for reporting community maternal deaths. Maternal death records should be available even at community level. Supervision should be intensified with all community stakeholders and HSAs on maternal death surveillance and response.

\section{Conclusion}

This study came up with important information on existing practices, knowledge, perceptions on reporting community maternal deaths in Mangochi district. Findings revealed that community maternal deaths were not reported to relevant authorities because records were not available for the past three years. Lack of knowledge was identified as the main reason for not reporting probably because participants were not trained. Participants' culture and religion did not prevent reporting community maternal deaths in Mangochi district.

\section{Conflict of Interest}

None of the authors had any conflict of interest in the manuscripts being published in this journal.

\section{Acknowledgements}

This study was funded by USAID as part of Master of Science Degree in Community Health Nursing for the senior author.

\section{References}

[1] Federal Democratic Republic of Ethiopia (2012) Maternal Death Surveillance and Response (MDSR) Technical Guideline. Ministry of Health,

[2] World Health Organisation (2007) Maternal Mortality in 2005: Estimates Developed by WHO, UNICEF, UNFPA and The World Bank.

[3] World Health Organisation (2012) Maternal Mortality. WHO, Geneva. http://www.who.int/mediacentre/factsheets/fs348/en/

[4] Nieburg, P. (2012) Improving Maternal Mortality and Other Aspects of Women's Health: The United States Global Role. A Report of the CSIS Global Health Policy Centre, Centre for Strategic \& International Studies, Washington DC.

[5] Kalter, H., Mohan, P., Mishra, A., Gaonkar, N., Biswas, A., Balakrishnan, S. and Babille, M. (2011) Maternal Death inquiry and Response in India-The Impact of Contextual Factors on Defining an Optimal Model to Help Meet Critical Maternal Health Policy Objectives. Health.

[6] Plata, N., Passano, P., Screenivas, A. and Gerdts, C. (2010) Maternal Mortality in Developing Countries: Challenges in Scaling-Up Priority Interventions Ndola Prata, Paige Passano, Amita Sreenivas \& Caitlin Elisabeth Gerdts. Women's Health, 2, 311-327.

[7] National Statistics Office (NSO) \& ICF Macro. (2011) Malawi Demographic and Health Survey 2010. NSO and ICF Macro, Zomba, Malawi, and Calverton, Maryland.

[8] Bakker, W., Akker, T., Mwagomba, B., Khukulu, R., Elteren, M. and Roosmalen, J. (2011) Health Workers’ Perceptions of Obstetric Critical Incident Audit in Thyolo District, Malawi. Tropical Medicine and International Health, 16, 1243-1250. http://dx.doi.org/10.1111/j.1365-3156.2011.02832.x

[9] Ratman, Y., Lungu, K., Hofman, J. and White, S. (2005) Why More Mothers Die: Confidential Inquiries into Institutional Maternal Deaths in the Southern Region of Malawi, 2001. Malawi Medical Journal, 17, 75-80.

[10] Hisar, N. and Sohoo, N. (2010) Maternal Mortality in Rural Community: A Challenge for Achieving Millennium Development Goal. Journal of Pakistan Medical Association, 60, 20-24.

[11] American Public Health Association (2011) Reducing US Maternal Mortality as a Human Right: Overview of Mater- 
nal Health as a Human Rights Issue in the United States. Washington DC.

[12] World Health Organisation (2004) Beyond the Numbers: Reviewing Maternal Deaths and Complications to Make Pregnancy Safer. Geneva.

[13] Mills, S., Lewis, G., Say, L., Mathews, M. and Okwero, P. (2011) Maternal Death Audit as a Tool Reducing Maternal Mortality.

[14] Kongnyuy, E., Mlava, G. and Broek, N. (2009) Facility-Based Maternal Death Review in Three Districts in the Central Region of Malawi: An Analysis of Causes and Characteristics of Maternal Deaths. Women's Health Issues: Official Publication of the Jacobs Institute of Women's Health, 19, 14-20. http://dx.doi.org/10.1016/j.whi.2008.09.008

[15] Hofman, J. and Ndemera, M. (2005) Review of Community Based Maternal Deaths in Mangochi District. Malawi Medical Journal, 17, 81-84.

[16] Ochi, H. (2012) Perceptions of Quality of Health Care Services and Maternal Mortality in Rural Communities in Kaduna State (Masters). Ahmadu Bello University, Zaria.

[17] United Nations Development Programme (2011) The Millennium Development Goals. New York.

[18] Groenewald, T. (2004) A Phenomenological Research Design Illustrated. International Journal of Qualitative Methods, 3, 1-26.

[19] Burns, N. and Grove, S. (2009) The Practice of Nursing Research: Appraisal, Synthesis, and Generation of Evidence. 6th Edition, Saunders, St. Louis.

[20] Colaizzi, P.F. (1978) Psychological Research as the Phenomenologist Views It. In: Valle, R.S. and King, M., Eds., Existential Phenomenological Alternatives for Psychology, Plenum, New York, 48-71.

[21] Edward, K. (2005) The Phenomenon of Resilience in Crisis Care Mental Health Clinicians. International Journal of Mental Health Nursing, 14, 142-148. http://dx.doi.org/10.1111/j.1440-0979.2005.00371.x

[22] Munjanja, S., Nystrom, L., Nyandoro, M. and Magwali, T. (2007) Maternal and Perinatal Mortality Study.

[23] Abdo, S., Jarrar, K., El-Nakhal, S., Ramlawi, A., Hijaz, T., Saman, K. and Abu-Fannonah, J. (2011) Report on Maternal Mortality in Palestine.

[24] Kalaichandran, A. and Zakus, D. (2007) The Obstetric Pathology of Poverty: Maternal Mortality in Kep Province, Cambodia. World Health and Population and World Health Organisation.

[25] McCaw-Binns, A., Lindo, J.L.M., Lewis-Bell, K.N. and Ashley, D.E.C. (2008) Maternal Mortality Surveillance in Jamaica. International Journal of Gynecology \& Obstetrics, 100, 31-36. http://dx.doi.org/10.1016/j.ijgo.2007.06.054

[26] Ali, M. and Kuroiwa, C. (2007) Accurate Record Keeping in Referral Hospitals in Pakistan's North West Frontier Province and Punjab: A Crucial Step Needed to Improve Maternal Health. Journal of Pakistan Med Association, 57, 443-446.

[27] Malawi Ministry of Health (2007) Road Map for Accelerating the Reduction of Maternal and Neonatal Mortality and Morbidity in Malawi. Ministry of Health, Lilongwe.

[28] Kululanga, L.I., Sundby, J., Malata, A. and Chirwa, E. (2011) Striving to Promote Male Involvement in Maternal Health Care in Rural and Urban Settings in Malawi-A Qualitative Study. Reproductive Health, 8, 36. http://dx.doi.org/10.1186/1742-4755-8-36

[29] Daniel, I., Graham, W. and Boerma, T. (2011) Maternal Death Surveillance and Response. Bulletin of the World Health Organization, 89, 779-779A. http://dx.doi.org/10.2471/BLT.11.097220

[30] Mairiga, A.G., Kawuwa, M.B. and Kulima, A. (2008) Community Perception of Maternal Mortality in Northeastern Nigeria. African Journal of Reproductive Health, 12, 27-34.

[31] Adah, S., Ogbonna, C., Anga, P., Chingle, M., Ashikeri, M., Envulandu, E. and Zoakah, A. (2009) The Impact of Advocacy and Community Mobilization on the Utilization of Health Services at the Comprehensive Health Centre.

[32] Lawoyin, T., Lawoyin, O., Olusheyi, O. and Adewole, D. (2007) Men’s Perception of Maternal Mortality in Nigeria. Journal of Public Health Policy, 28, 299-318. http://dx.doi.org/10.1057/palgrave.jphp.3200143 


\section{Abbreviations}

DHO: District Health Officer;

FGD: Focus Group Discussions;

HAS: Health Surveillance Assistant;

MDHS: Malawi Demographic Health Survey;

COMREC: College of Medicine Research and Ethics Committee;

WHO: World Health Organization. 